\title{
Radial dependence of foreshock cavities: a case study
}

\author{
D. G. Sibeck ${ }^{1}$, K. Kudela ${ }^{2}$, T. Mukai ${ }^{3}$, Z. Nemecek ${ }^{4}$, and J. Safrankova ${ }^{4}$ \\ ${ }^{1}$ NASA Goddard Space Flight Center, Greenbelt, MD, 20771, USA \\ ${ }^{2}$ Institute of Experimental Physics Slovak Academy of Sciences, Kosice, Slovakia \\ ${ }^{3}$ Institute of Space and Astronautical Sciences, Kanagawa, Japan \\ ${ }^{4}$ Charles University, Faculty of Mathematics and Physics, Prague, Czech Republic
}

Received: 13 July 2004 - Revised: 28 September 2004 - Accepted: 6 October 2004 - Published: 22 December 2004

\begin{abstract}
We present a case study of Geotail, Interball-1, IMP-8, and Wind observations of density and magnetic field strength cavities excavated by the enhanced pressures associated with bursts of energetic ions in the foreshock. Consistent with theoretical predictions, the pressure of the energetic ions diminishes rapidly with upstream distance due to a decrease in the flux of energetic ions and a transition from near-isotropic to streaming pitch angle distributions. Consequently, the cavities can only be observed immediately upstream from the bow shock. A comparison of conditions upstream from the pre- and post-noon bow shock demonstrates that foreshock cavities introduce perturbations into the oncoming solar wind flow with dimensions smaller than those of the magnetosphere. Dayside geosynchronous magnetic field strength variations observed by GOES-8 do not track the density variations seen by any of the spacecraft upstream from the bow shock in a one-to-one manner, indicating that none of these spacecraft observed the precise sequence of density variations that actually struck the subsolar magnetopause.
\end{abstract}

Key words. Interplanetary physics (energetic particles; planetary bow shocks) - Magnetospheric physics (solar wind-magnetosphere interactions)

\section{Introduction}

Kinetic processes occurring in the immediate vicinity of the Earth's bow shock can introduce considerable structure into the solar wind shortly prior to its interaction with the Earth's magnetosphere. The relatively rare hot flow anomalies (Schwartz et al., 1985) and much more common foreshock cavities (e.g. Sibeck et al., 2002) represent prominent examples. The latter structures form on bundles of magnetic field lines connected to the bow shock that are embedded in regions of magnetic field disconnected from the bow shock. The bundles fill with enhanced fluxes of suprathermal ions generated at the bow shock. The pressure of these ions

Correspondence to: D. G. Sibeck

(david.g.sibeck@nasa.gov) perpendicular to the magnetic field causes the bundles to expand, resulting in cavities of depressed density and magnetic field strength bounded by regions of enhanced densities and magnetic field strengths.

We can use the predictions of the Fermi (e.g. Terasawa, 1979; Lee, 1982) and reflection (Sonnerup, 1969) models for the ion foreshock to determine where foreshock cavities should be most prominent. As illustrated in Fig. 1, models for Fermi acceleration predict intense, nearly isotropic, fluxes of suprathermal ions with energies extending beyond $300 \mathrm{keV}$ immediately upstream from the quasi-parallel bow shock on magnetic field lines that have been connected to the bow shock for many minutes $\left(t_{3}\right)$. Lower fluxes and particle distributions streaming away from the bow shock occur both further upstream and on magnetic field lines only recently connected to the bow shock. Models for ion reflection from the quasi-perpendicular bow shock (Sonnerup, 1969) predict highly anisotropic beams of suprathermal $(\sim 4 \mathrm{keV})$ ions streaming along interplanetary magnetic field (IMF) lines recently connected to the bow shock $\left(t_{2}\right)$. No energetic ions are present far upstream or on IMF field lines unconnected to the bow shock $\left(\mathrm{t}_{1}\right)$. Numerous observational studies confirm these predictions (e.g. Gosling et al., 1978; Greenstadt et al., 1980; Scholer et al., 1980). Other studies indicate that fluxes also increase as $\Theta_{B n}$ decreases (Mitchell and Roelof, 1983), where $\Theta_{B n}$ is the angle between individual IMF lines and the normal to the bow shock at the point of intersection.

Based on these predictions, foreshock cavities should be most prominent immediately upstream from the pre-noon bow shock during typical periods of spiral IMF orientation. As illustrated in Fig. 1, field lines at pre-noon local times have been connected to the bow shock longer than field lines connected to the post-noon bow shock. Furthermore, $\Theta_{B n}$ is less for field lines connected to the pre-noon foreshock than for those connected to the post-noon bow shock.

To test the proposed foreshock cavity scenario, we present a case study of simultaneous foreshock observations by three ISTP-era spacecraft upstream from the post-noon bow shock and one spacecraft upstream from the pre-noon bow shock. During this interval, the IMF generally assumed an unusual, orthospiral orientation. The three spacecraft upstream from 


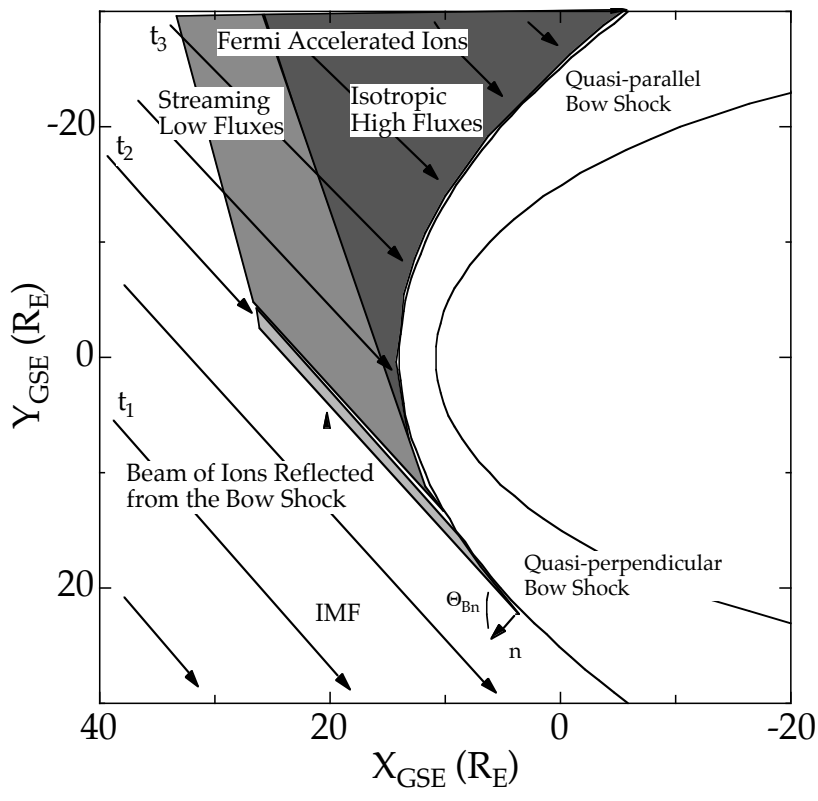

Fig. 1. Spatial distribution of suprathermal ion distributions in the foreshock. No suprathermal ions occur on magnetic field lines unconnected to the bow shock $\left(t_{1}\right)$. A beam of reflected or leaking ions can be found on magnetic field lines connected to the quasiperpendicular bow shock $\left(t_{2}\right)$. A high flux of nearly isotropic ions can be found immediately upstream from the quasi-parallel bow shock. A lower flux of streaming ions can be found further upstream $\left(t_{3}\right)$.

the post-noon bow shock observed corresponding foreshock cavities. The amplitudes of the density and magnetic field strength perturbations associated with these cavities diminished with distance from the bow shock. As predicted by the model described above, the flux of suprathermal ions also diminished with distance upstream and the particle pitch angle distributions became more field-aligned. By contrast, neither the length of time a field line had been connected to the bow shock nor $\Theta_{B n}$ were important factors in determining event strength. The spacecraft outside the pre-noon bow shock observed completely different energetic ion bursts, foreshock cavities, and density and magnetic field strength variations. As a result, the solar wind did not apply a uniform pressure across the entire dayside magnetosphere. In fact, a comparison of the upstream solar wind observations with those by GOES- 8 of the dayside magnetospheric magnetic field strength indicates that none of the solar wind monitors observed the precise sequence of pressure variations that buffeted the subsolar magnetopause.

\section{Data sets}

To consider the radial dependence of the properties describing foreshock cavities, we will present simultaneous Geotail, Interball-1, IMP-8, and Wind solar wind plasma, magnetic field, and energetic ion observations. From Geotail, we will present LEP plasma density observations with 12.2- s time resolution (Mukai et al., 1994), MGF magnetic field strength observations with 3-s time resolution (Kokubun et al., 1994), and EPIC energetic ion flux observations with 6-s time resolution (Williams et al., 1994). From Interball-1, we will present VDP plasma flux observations with 15-s time resolution (Safrankova et al., 1997), MIF-M magnetic field strength observations with 6-s time resolution (Klimov et al., 1997), and DOK-2 energetic ion observations with a variable time resolution that depended on energetic ion flux levels (Lutsenko et al., 1998). The IMP-8 MIT Faraday cup takes snapshots of the density over $21.6 \mathrm{~s}$ at a maximum rate of once each $58 \mathrm{~s}$. We present IMP-8 magnetic field observations with a time resolution of $6 \mathrm{~s}$ and EPE energetic ion observations with $21.6 \mathrm{~s}$ time resolution (King, 1982). From Wind, we will present 3-DP plasma and energetic ion observations with 3- and 6-s time resolution, respectively (Lin et al., 1995), and MGI magnetic field strength observations with 3-s time resolution (Lepping et al., 1995). To describe the magnetospheric response, we will present GOES-8 geosynchronous magnetometer observations with 1-min time resolution (Singer et al., 1996).

\section{Solar wind observations}

We present observations for the period from 16:00-19:00 UT on 19 April 1996. As shown in Fig. 2, during this interval Geotail moved duskward from Geocentric Solar Ecliptic $(\mathrm{GSE} x, \mathrm{y}, \mathrm{z})=(20.0,6.0,-1.9)$ to $(20.4,8.8,-2.2)$ Earth radii $\left(\mathrm{R}_{E}\right)$ immediately upstream from the post-noon bow shock. Interball-1 moved equatorward and earthward from $\operatorname{GSE}(\mathrm{x}, \mathrm{y}, \mathrm{z})=(23.2,15.0,4.1)$ to $(21.9,14.4,2.3) \mathrm{R}_{E}$ from a location somewhat further upstream and outside the equatorial post-noon bow shock. IMP-8 moved duskward from $\operatorname{GSE}(\mathrm{x}, \mathrm{y}, \mathrm{z})=(30.6,17.3,17.6)$ to $(29.4,20.1,16.3) \mathrm{R}_{E}$ from a position still further upstream and outside the northern post-noon bow shock. Wind moved sunward from GSE (x, y, $\mathrm{z})=(5.4,-26.3,2.4)$ to $(9.9,-28.3,2.5) \mathrm{R}_{E}$ immediately outside the equatorial dawn bow shock. Also shown in Figure 2 are the nominal positions of the Earth's bow shock (Fairfield, 1971) and magnetopause (Roelof and Sibeck, 1993). GOES8 moved through the dayside magnetosphere and local noon from GSE $(\mathrm{x}, \mathrm{y}, \mathrm{z})=(6.3,-2.0,-2.2)$ to $(5.6,2.7,-2.2)$ along its geosynchronous orbit.

Figure 3 summarizes Geotail, Interball-1, IMP-8, and Wind plasma, magnetic field, and energetic ion observations during the three-hour interval on 19 April 1996. The EPIC instrument on Geotail observed one brief and three prolonged energetic (61.5-73.7 keV) ion bursts: from 16:05 to 16:07, $16: 16$ to $16: 39,16: 59$ to $17: 29$, and $17: 44$ to $18: 13$ UT. During these bursts, Geotail observed correlated large amplitude plasma density and magnetic field strength variations. The most prominent peaks in the energetic ion flux corresponded to density and magnetic field strength minima (e.g. at 16:07, 16:22, 16:26, 16:29, 17:06, 17:19, 17:26, and 17:54 UT). Consequently, these minima can be interpreted as foreshock cavities excavated by the ion bursts. 


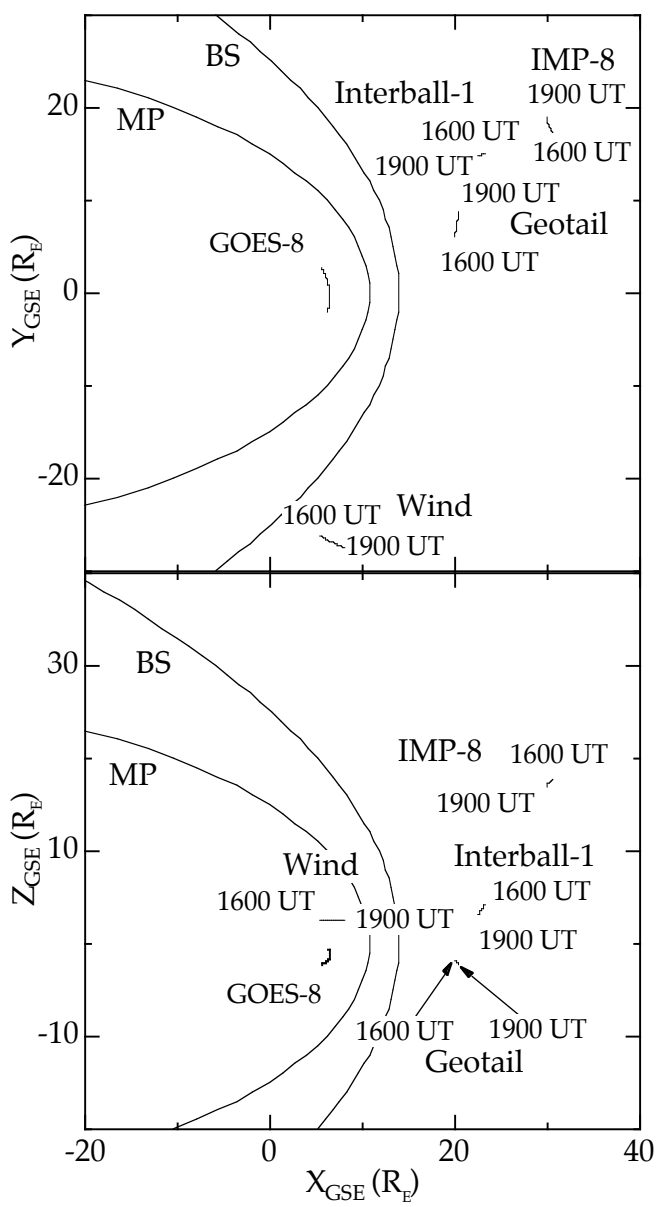

Fig. 2. Ecliptic and meridional projections of Geotail, Interball-1, IMP-8, Wind, and GOES-8 trajectories from 16:00 to 19:00 UT on 19 April 1996. For comparison, the figure also shows the nominal positions of the bow shock and magnetopause.

By contrast to the large amplitude fluctuations that occurred during intervals when energetic ions were present, Geotail observed relatively constant densities and magnetic field strengths during intervals when the flux of energetic ions fell to background levels, e.g. from 16:40 to 17:00 and after 18:10 UT. The modest density and magnetic field strength variations during these intervals were in antiphase (e.g. 18:30 to 19:00 UT), as is generally the case for intrinsic solar wind tangential discontinuities (e.g. Burlaga, 1968).

Now consider the Interball-1 observations, made several $\mathrm{R}_{E}$ further upstream. Interball-1 observed bursts of enhanced energetic $(46.3-60 \mathrm{keV})$ ion fluxes from 16:13 to 16:40 UT, $16: 52$ to $17: 36$, and 17:52 to $18: 12$ UT, corresponding to the three prolonged bursts at Geotail. As in the case of Geotail, Interball-1 observed correlated magnetic field strength and plasma flux $(\mathrm{nV})$ variations during each of these intervals. However, the amplitudes of these perturbations were lower than those seen at Geotail. Also like Geotail, Interball-1 observed weak antiphase plasma flux and magnetic field strength variations during intervals when no energetic ions were present (e.g. from 18:30 to 19:00 UT).

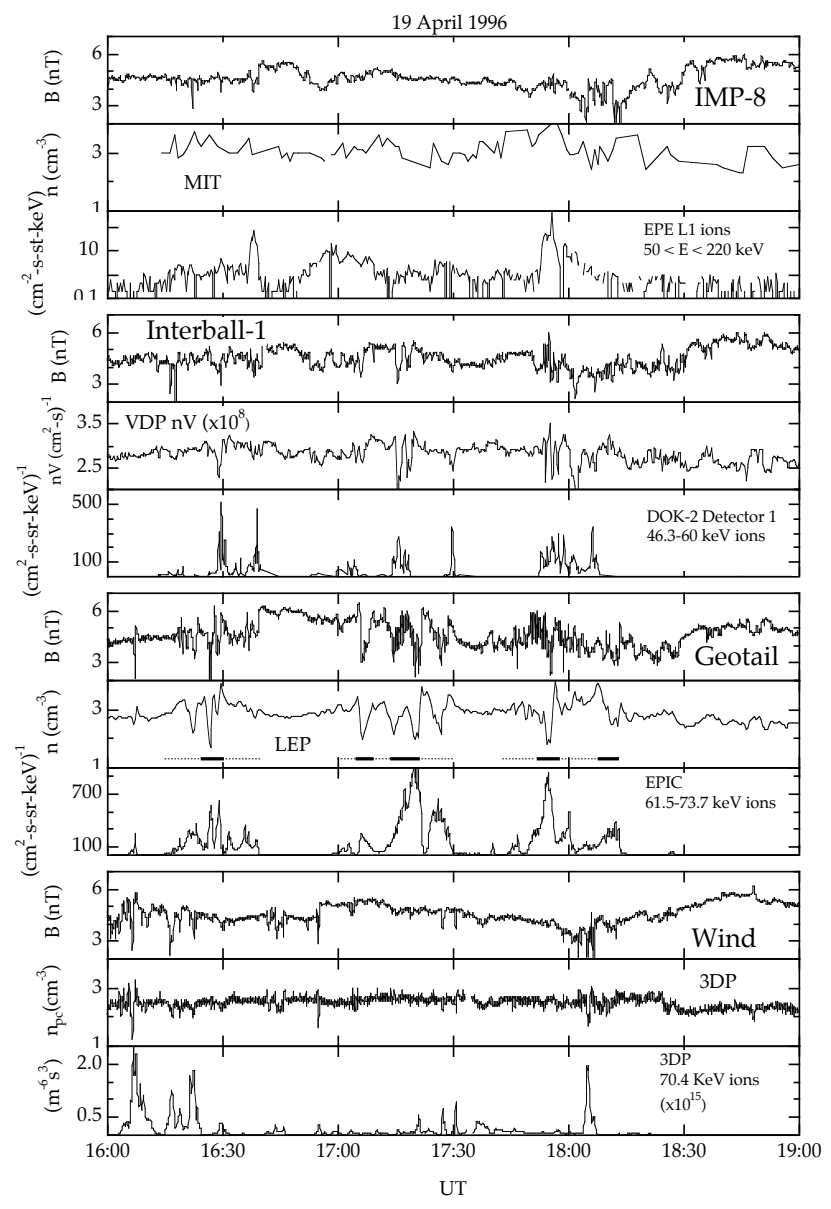

Fig. 3. A summary plot of IMP-8, Interball-1, Geotail, and Wind solar wind and foreshock observations from 16:00-19:00 UT on 19 April 1996. The first triplet of panels depicts the IMP-8 GSFC magnetic field strength, MIT ion plasma density, and EPE energetic ion observations. The second triplet of panels shows Interball-1 MIF-M magnetic field strength, VDP ion plasma flux, and DOK-2 energetic ion flux observations. The third triplet of panels shows Geotail MGF magnetic field strength, LEP ion plasma density, and EPIC energetic ion flux observations. The fourth triplet of panels shows Wind MGI magnetic field strength, 3-DP ion plasma density, and 3-DP energetic ion phase space density observations.

Located even further upstream, IMP-8 observed three bursts of energetic (50-220 keV) ions, from 16:17 to $16: 39$, 16:50 to 17:33, and 17:52 to 18:11 UT, again corresponding to the three bursts at Geotail. The time resolution of the IMP8 plasma observations does not suffice to determine whether decreases in the density corresponded to bursts of energetic ions or whether the density and magnetic field perturbations were in phase or antiphase. However, because the energetic ion bursts did not correspond to decreases in the magnetic field strength, the bursts at IMP-8 cannot be interpreted as foreshock cavities. Note, however, that there was a small $(\sim 1 \mathrm{nT})$ decrease in the IMP-8 magnetic field strength when the energetic ion flux peaked at 16:38 UT. 


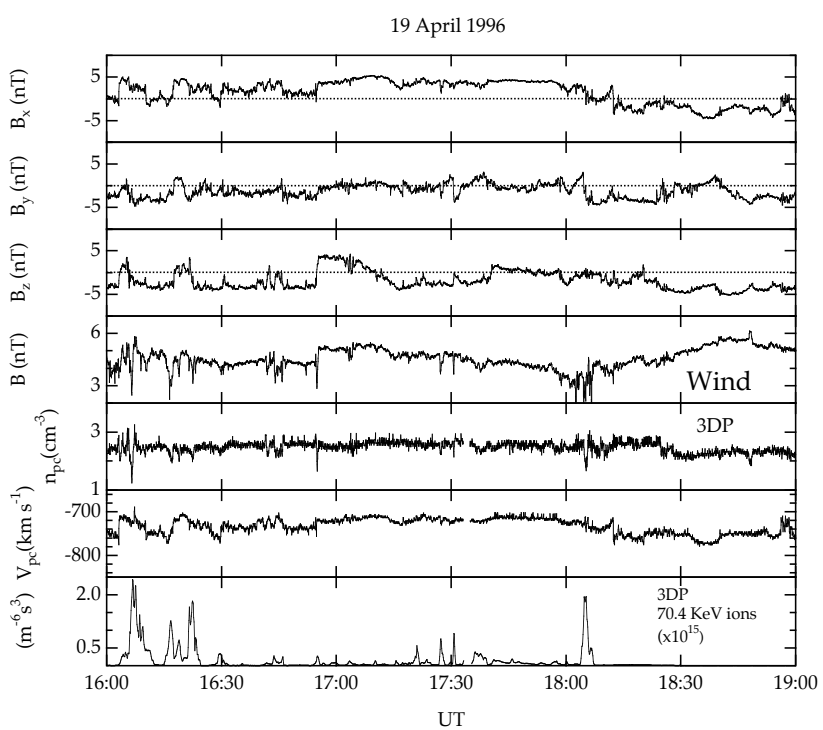

Fig. 4. Wind MGI observations of the IMF strength and direction (GSE coordinates), 3-DP ion plasma density, velocity along the Sun-Earth line, and energetic ion phase space density observations. Wind observes energetic ions during intervals when the IMF connects the spacecraft to the bow shock, i.e. when the IMF points sunward and dawnward.

We can use Wind observations to demonstrate that the bursts of energetic ions are observed during intervals when the IMF connects each spacecraft to the bow shock. During the three-hour interval shown in Fig. 4, Wind was located upstream from the pre-noon bow shock. A sunward and dawnward IMF orientation should connect the spacecraft to that boundary. As indicated in Fig. 4, Wind observed energetic ions almost continuously from 16:00 to 18:30 UT, an interval of sunward $\left(+B_{x}\right)$ and dawnward $\left(-B_{y}\right)$ IMF orientation. By contrast, fluxes fell to background levels after 18:30 UT, when the IMF pointed antisunward and dawnward, and did not connect the spacecraft to the bow shock. Note also that the solar wind velocity varied by no more than $5 \%$ about a mean value of $\sim 730 \mathrm{~m} \mathrm{~s}^{-1}$. Any plasma flux or dynamic pressure variations on this day resulted primarily from density, and not velocity, fluctuations.

We wish to determine whether the differing amplitudes of the perturbations associated with the foreshock cavities observed by Geotail, Interball-1, and IMP-8 are consistent with the model for foreshock cavities described above. To do so, we should first demonstrate that pitch angle distributions were more field-aligned at IMP-8 than Interball-1, and more field-aligned at Interball-1 than Geotail. We should then show that flux levels were lower at IMP-8 than Interball1 , and at Interball-1 than Geotail.

Figure 5 provides information about the energetic ion pitch angle distributions observed by Geotail, Interball-1, and IMP-8. In combination, the northern and southern heads of the EPIC instrument on Geotail provided good pitch angle coverage throughout this interval. Geotail observed nearly isotropic energetic (52.7 to $61.5 \mathrm{keV})$ ion pitch

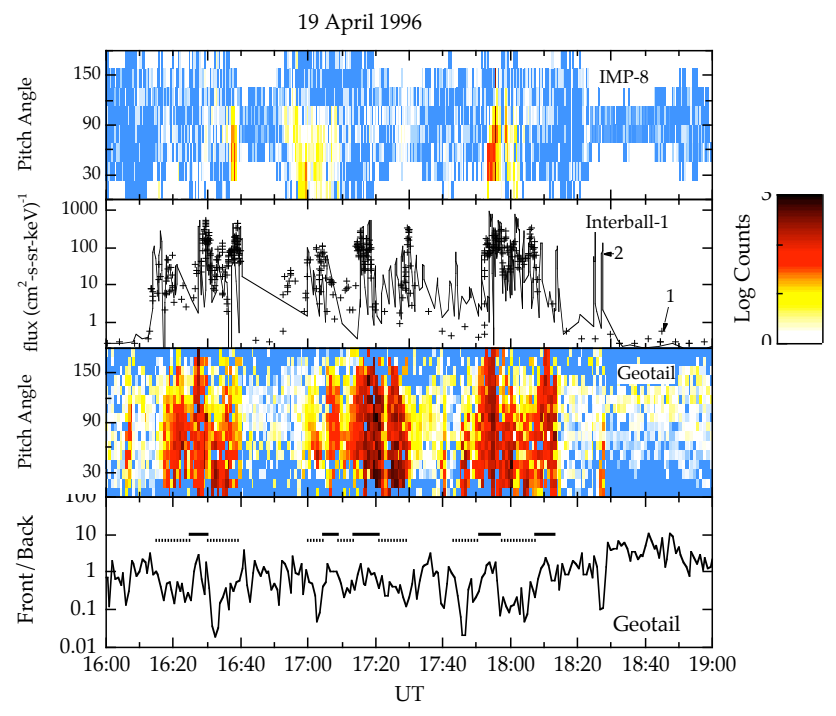

Fig. 5. IMP-8, Interball-1, and Geotail energetic ion pitch angle distributions. The top panel shows pitch angle distributions for the L1 (50-220 keV) channel on IMP-8. The second panel compares traces for the antisunward (Detector 1, crosses) and spinning (Detector 2, solid curve) sensors of the DOK-2 instrument on Interball-1. Detector 1 observed ions with energies from 46.3 to $60 \mathrm{keV}$, whereas Detector 2 observed ions with energies from 48.2 to $59 \mathrm{keV}$. The third panel shows Geotail pitch angle distributions for 52.7 to $61.5 \mathrm{keV}$ ions. It was obtained by combining observations from both heads. The bottom panel shows Geotail front to back ratios for the 52.7 to $61.5 \mathrm{keV}$ ions, defined as the ratio of the ion flux with pitch angles ranging from 0 to $90^{\circ}$ divided by the flux with pitch angles ranging from 90 to $180^{\circ}$.

angle distributions and small front to back ratios from 16:25$16: 30$ UT, $17: 05$ to $17: 09,17: 14$ to $17: 21,17: 52$ to $17: 57$ and $18: 08$ to $18: 13$ UT (solid bars), but broad beams were streaming away from the bow shock along the magnetic field and large front to back ratios from $16: 15$ to $16: 25,16: 30$ to $16: 40,17: 00$ to $17: 05,17: 09$ to $17: 14,17: 21$ to $17: 30,17: 43$ to $17: 51$, and 17:57 to 18:08 UT (dashed bars). These horizontal bars are repeated in the LEP density panel of Fig. 3 (and other figures in this paper) to demonstrate that the intervals of near-isotropic fluxes correspond to energetic ion flux enhancements, and density and magnetic field strength minima.

The EPE instrument on IMP-8 also provided relatively good pitch angle coverage. By contrast to Geotail, IMP-8 never recorded energetic ( 50 to $220 \mathrm{keV}$ ) ions over the full range of pitch angles observed. Instead, it only observed strongly anisotropic bursts of energetic ions streaming sunward along the magnetic field at 16:30 UT and from 16:50 to 17:10 and 17:50 to 18:02 UT. The absence of any foreshock cavities at IMP-8 (Fig. 3) indicates that the pressures associated with these anisotropic distributions failed to excavate any cavities.

Although the DOK-2 instrument on Interball-1 did not provide full pitch angle coverage, some inferences 
19 April 1996 (1754 UT)

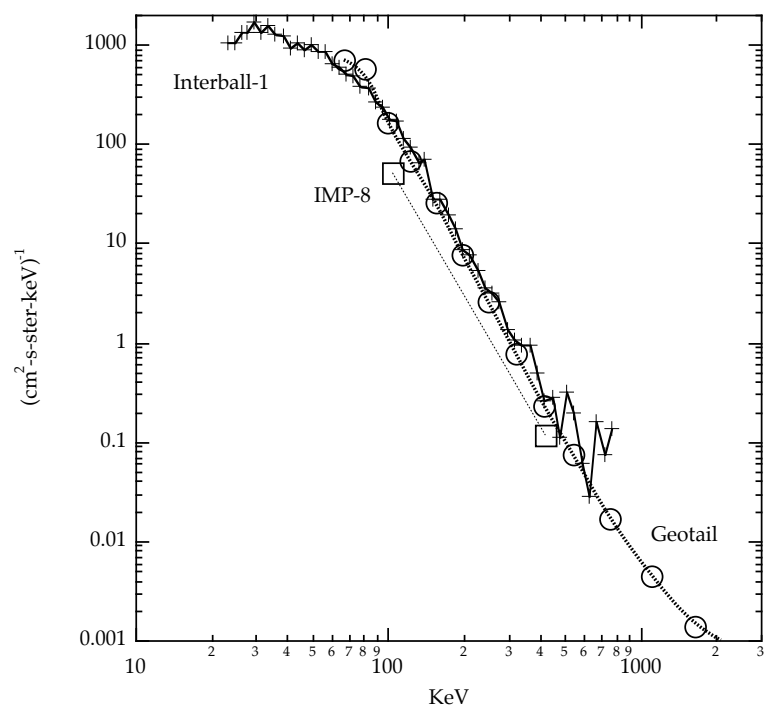

Fig. 6. A comparison of Geotail (circles), Interball-1 (crosses, and IMP-8 (squares) energetic ion spectra near 17:54 UT on 19 April 1996. The spectra were selected when the flux peaked: 17:53:17 UT on Geotail, 17:56:39 UT at Interball, and 17:53:55 UT at IMP-8.

concerning energetic ion anisotropies at Interball-1 can be obtained by comparing the observations made by its two detectors. Whereas Detector 1 pointed directly antisunward, Detector 2 pointed $62.5^{\circ}$ away from the sunward-oriented spin axis. When the two detectors observe nearly identical flux levels, and Detector 2 observes no spin modulation, we may infer nearly isotropic particle distributions. The observations shown in Fig. 5 indicate that these conditions were generally not met, i.e. that significant anisotropies were usually present. However, the energetic $(\sim 47$ to $\sim 60 \mathrm{keV})$ ion fluxes approached isotropy at 16:30, 16:40, 17:15-17:20, and 17:53 to 18:02 UT, precisely the times when Fig. 3 indicates the in-phase plasma flux and magnetic field strength variations that identify cavities at Interball-1.

Having shown that pitch angle distributions were often more isotropic at Geotail and Interball-1 than at IMP-8, we now wish to determine whether energetic ion flux levels were higher at Geotail than Interball-1 and higher at Interball-1 than IMP-8. Figure 6 compares energetic ion spectra observed by Geotail, Interball-1 (crosses), and IMP-8 (squares) near 17:54 UT at the moment when fluxes peaked at each spacecraft. The comparison indicates that fluxes at Interball1 reached levels similar to those at Geotail, but that those at IMP-8 were a factor of 3 lower. Over the range of energies from 67.3 to $154 \mathrm{keV}$, the Geotail spectra is best fit by an exponential, $\mathrm{f}=\mathrm{f}_{0} \mathrm{e}^{-E / E 0}$, with an e-folding energy of $\mathrm{E}_{0}=26 \mathrm{keV}$.

To decide whether the observations at 17:54 UT typified those for the entire three-hour interval, we must compare ion fluxes at similar energies. Figure 3 presented IMP- 8 observations of 50-220 keV ions, Interball-1 observations of 46.3$60 \mathrm{keV}$ ions, and Geotail observations of $61.5-73.7 \mathrm{keV}$ ions.

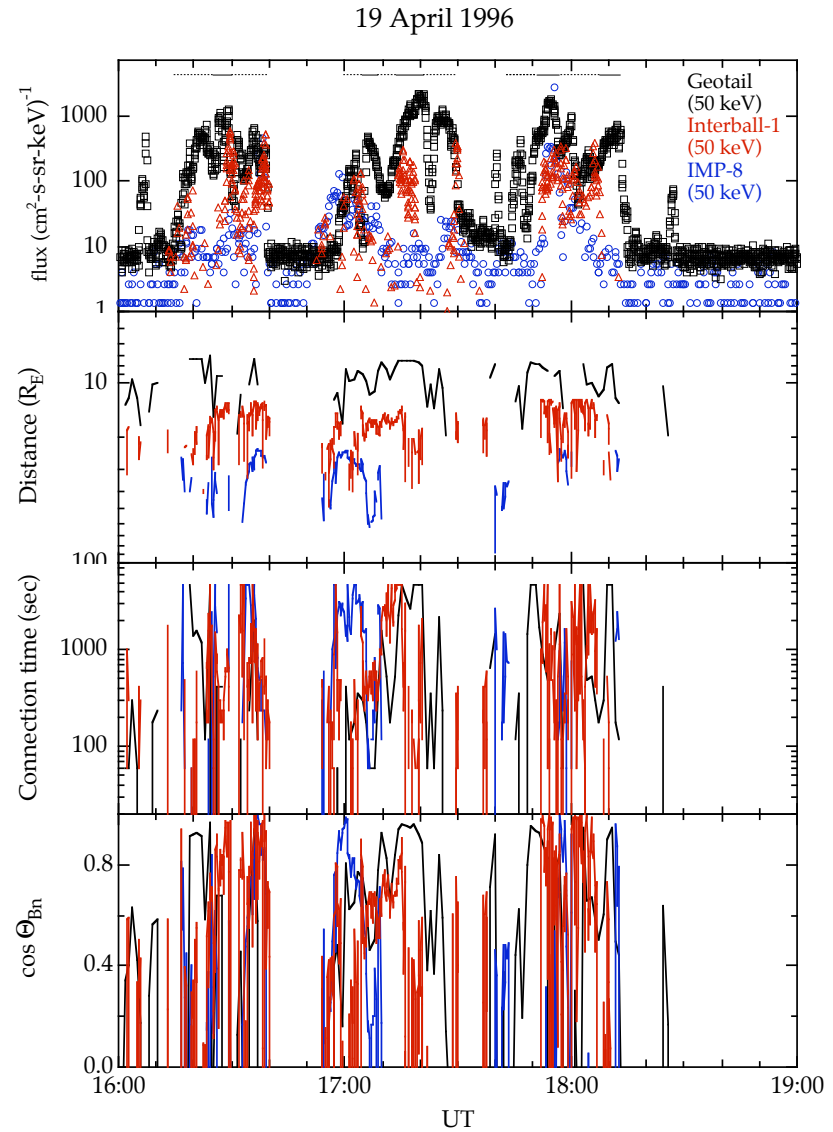

Fig. 7. A comparison of Geotail, Interball-1, and IMP-8 energetic ion observations with factors thought to control flux levels. The top panel presents energetic ion flux levels observed by Geotail (61.5$73.7 \mathrm{keV})$, Interball-1 (46.3-60 keV), and IMP-8 (50-220 keV). For comparison, the flux levels have been extrapolated to those expected at $50 \mathrm{keV}$ under an assumption of an exponential spectra with an efolding energy of $26 \mathrm{keV}$. The second panel presents the distances of each spacecraft from the bow shock along the IMF. The third panel depicts the length of time each field line had been connected to the bow shock when it encountered the observing spacecraft. The bottom panels presents $\cos \Theta_{B n}$, the angle between the IMF field line connecting observing spacecraft to the bow shock and the normal to the bow shock at the point of intersection.

The top panel of Fig. 7 compares the same observations, but at energies of $50 \mathrm{keV}$, assuming exponential spectra with an e-folding energy of $26 \mathrm{keV}$. Despite flux variations greater than two orders of magnitude at each spacecraft, fluxes at Interball-1 were almost invariably less than those at Geotail, while those at IMP-8 were almost invariably less than those at Interball-1. We conclude that the ordering of the observations at 17:54 UT typifies that for the entire three-hour interval.

We now wish to determine whether the systematic differences in energetic ion flux levels, energetic ion pitch angle distributions, and plasma and magnetic field perturbation amplitudes seen by IMP-8, Interball-1, and Geotail primarily result from: 1) different distances of the three spacecraft along 


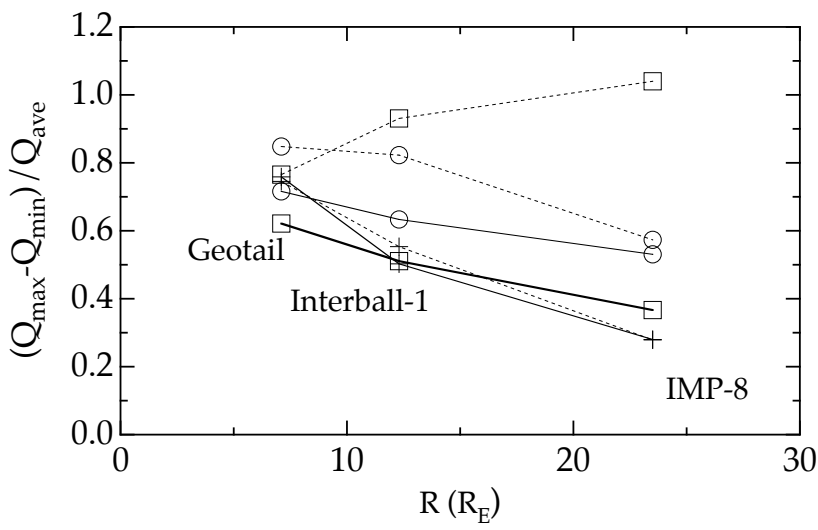

Fig. 8. Normalized values for the density (solid lines segments) and magnetic field strength (dashed line segments) variations as a function of distance along the IMF from the bow shock during three time intervals 16:00-16:40 (crosses), 16:50 to 17:35 (circles), and $17: 40$ to $18: 15$ (squares) UT.

the IMF to the bow shock, 2) different bow shock connection times for the IMF field lines within the events, or 3) different $\Theta_{B n}$ at the intersection of the IMF lines with the bow shock.

The lower three panels of Fig. 7 provide the information needed to test these hypotheses. As can be seen in the second panel of Fig. 7, the distance from IMP-8 to the model bow shock (Fairfield, 1971) along the IMF was invariably greater than that for Interball-1, while Interball-1 was invariably further than Geotail. We have assumed that there are no kinks in the IMF lines between the spacecraft and the bow shock, and no values for the distance are shown when the IMF did not connect the spacecraft to the model bow shock. Consequently, the distance from the bow shock along the IMF provides a good explanation for the transition from high flux levels and isotropic ion distributions at Geotail, to low flux levels and anisotropic distributions at IMP-8.

Now consider the two alternatives, namely that energetic ion flux levels and pitch angle distributions are determined primarily by the length of time field lines are connected to the bow shock or by $\Theta_{B n}$ at the point the field lines intersect the bow shock. To calculate the length of time a field line has been connected to the bow shock, we simply convect the line with the observed solar wind speed at 1-min time steps from the point of its initial connection, where it lies tangent to the bow shock, until it reaches the location of the observing spacecraft. As can be seen in the third panel of Fig. 7, the field lines observed by Geotail were not systematically connected to the bow shock longer than those observed by Interball-1, nor were those observed by Interball-1 systematically connected longer than those observed by Geotail. In addition, as shown in the bottom panel of Fig. 7, there were no systematic differences in the angles between the IMF lines observed by the spacecraft and the normal to the model bow shock (Fairfield, 1971) at their point of intersection.

Consequently, we conclude that the primary factor controlling flux levels and pitch angle distributions in the region upstream from the bow shock is the distance of the observing spacecraft from the bow shock along the IMF lines. Figure 8 presents the normalized peak-to-peak amplitude of the density/plasma flux (solid lines) and magnetic field strength (dashed lines) variations at Geotail, Interball-1, and IMP-8, as a function of distance from the bow shock during three energetic ion burst intervals: 16:00 to 16:40 (crosses), 16:50 to $17: 35$ (circles), and 17:40 to 18:15 UT (boxes). Geotail magnetic field observations were averaged to $6 \mathrm{~s}$ for this plot. Density/plasma flux observations were averaged to 24-, 15-, and 21.6-s time resolution for Geotail, Interball-1, and IMP-8, respectively. The peak-to-peak amplitudes were normalized by the mean values during each interval, and the values were plotted at the value for the minimum distance connecting each spacecraft to the bow shock. With one exception, the figure clearly demonstrates the predicted decrease in plasma and magnetic field perturbation amplitudes with distance upstream from the bow shock. The exception occurs during the interval from 17:40-18:15 UT, during which IMP8 observed considerably larger magnetic field strength variations than Interball-1, and Interball-1 observed larger magnetic field strength variations than Geotail. These variations were apparently caused by very weak fields at the former spacecraft that went unobserved by Geotail.

\section{Pre-noon foreshock and magnetospheric observa- tions.}

Neither IMP-8 nor Interball-1, nor even Geotail, observed the precise sequence of solar wind or foreshock-modified plasma and magnetic field parameters that actually struck the dayside magnetosphere. To demonstrate this, we compare post-noon observations of the IMF orientation by IMP-8, Interball-1, and Geotail with pre-noon observations by Wind. We then compare observations of the solar wind input by each of these four spacecraft with observations of the magnetospheric response by GOES-8.

Figure 9 presents observations of IMF $B_{z}$ by IMP-8, Interball-1, Geotail, and Wind in GSE coordinates. As they were all located upstream from the post-noon bow shock, one would expect IMP-8, Interball-1, and Geotail to observe similar solar wind features. In broad terms, they did observe a gradual southward rotation from 16:50 to 17:15 UT, following an abrupt northward turning shortly after 16:30 UT. However, close inspection reveals some discrepancies on shorter time scales. For example, IMP-8 observed two intervals of northward IMF prior to 16:30 UT, Interball-1 three intervals but Geotail only one interval.

As Wind was located upstream from the dawn bow shock, we expect its observations to differ from those of IMP-8, Interball-1, and Geotail. Figure 9 confirms that this was indeed the case. While Wind observed an abrupt northward turning at 16:55 UT, it also observed a much more rapid subsequent rotation back to southward IMF orientations than any of the other spacecraft. The preceding northward turning(s), so prominent at the other spacecraft, were far less 
19 April 1996

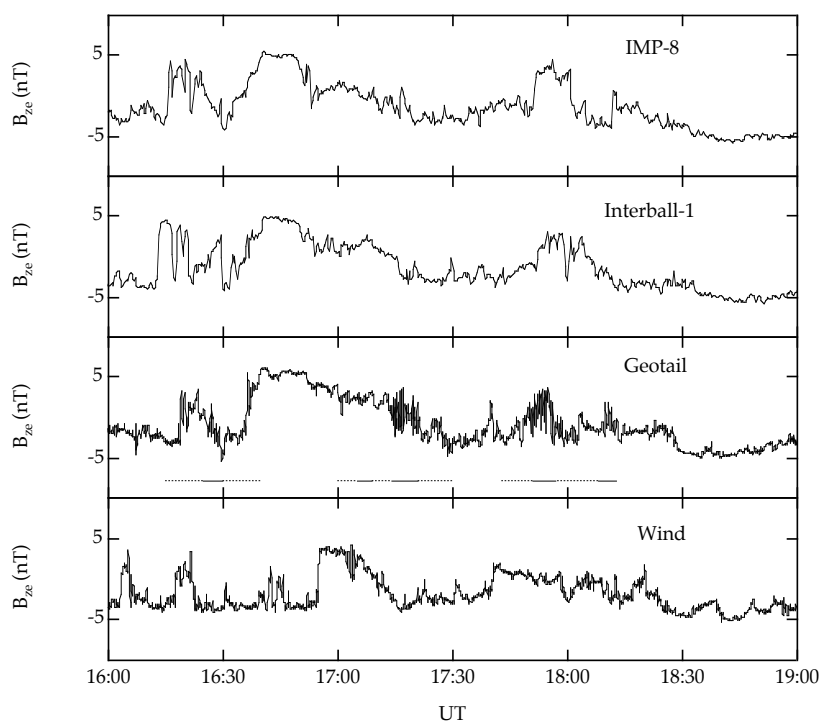

Fig. 9. IMP-8, Interball-1, Geotail, and Wind observations of IMF $B_{z}$ in GSE coordinates from 16:00-19:00 UT on 19 April 1996.

pronounced at Wind. Some or all of these discrepancies may result from the differing lag times required for solar wind features to move from one spacecraft to another (Weimer et al., 2002).

We conclude that during the interval under study, the pristine IMF structure, unperturbed by processes within the foreshock, varied over distances comparable to the dimensions of the magnetosphere.

However, the processes that occur within the foreshock introduce far greater spatial variations, at least in the plasma parameters that batter the magnetosphere. IMF orientations that connected IMP-8, Interball-1, and Geotail to the bow shock did not connect Wind, and vice versa. Consequently, foreshock cavities generated at the first three spacecraft cannot correspond to those at the latter. Returning to Fig. 3, we note that Wind observed a pattern of magnetic field strength, density, and energetic ion phase space density variations that differs from those seen by the other spacecraft. In particular, with the exception of two very transient foreshock cavities at 16:06 and 18:05 UT that occurred in response to strong bursts of energetic ions, Wind observed far steadier magnetic field strengths and densities than either Interball-1 or Geotail. Nevertheless, Wind did observe prominent foreshock cavities later on this day when the IMF connected it to the bow shock (Sibeck et al., 2002).

Pressure variations generated within the foreshock, and those intrinsic to the solar wind, launch fast mode waves when they strike the bow shock (Thomas and Brecht, 1988). Because the sum of the fast mode and magnetosheath velocities is approximately equivalent to that of the solar wind itself, pressure fronts in the magnetosheath keep pace with those in the solar wind. Consequently, the sequence of pressure variations striking the magnetopause should correspond

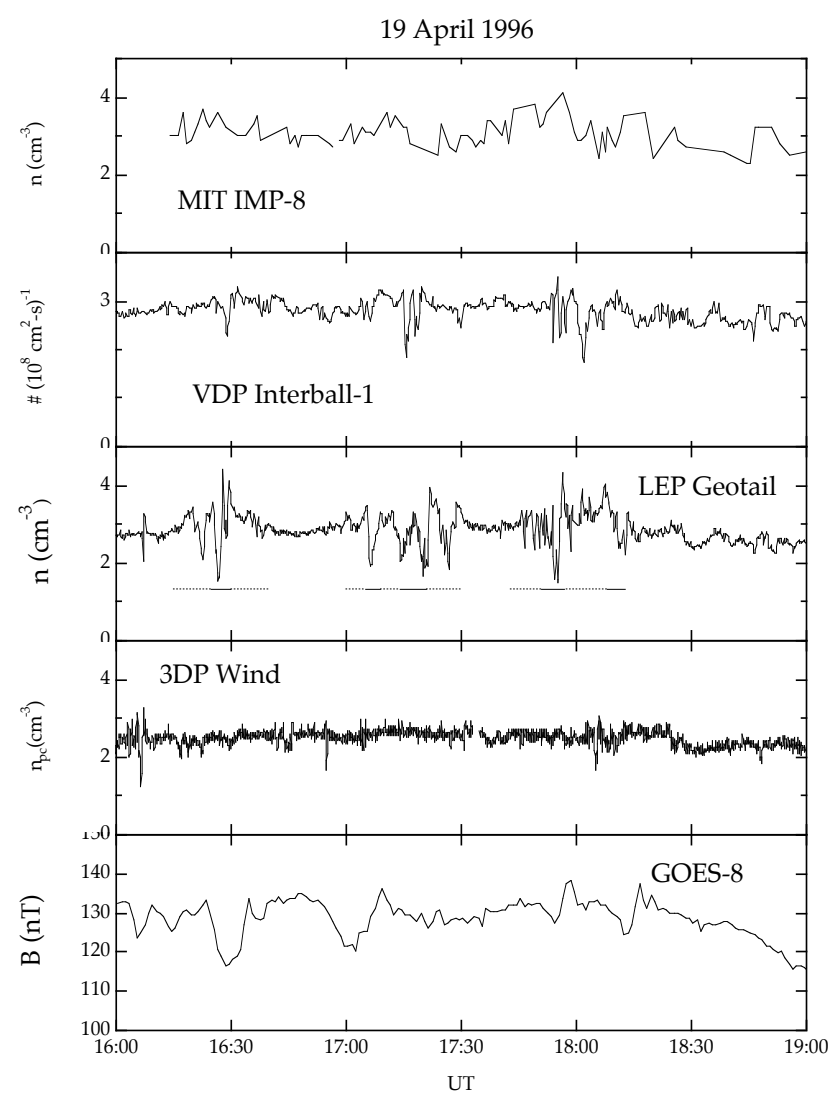

Fig. 10. A comparison of the solar wind density observed by IMP-8, the solar wind flux (nV) observed by Interball-1, the solar wind density observed by Geotail, the solar wind density observed by Wind, and the dayside geosynchronous magnetic field strength observed by GOES-8 from 16:00 to 19:00 UT on 19 April 1996.

to that of solar wind density variations incident on the bow shock. Each pressure variation striking the magnetopause should, in turn, launch a fast mode wave into the magnetosphere. Thus, observations by dayside geosynchronous spacecraft provide an opportunity to determine which, if any, of the solar wind monitors available on this day observed the precise sequence of density and pressure variations that actually struck the magnetosphere. Past work indicates that the dayside geosynchronous magnetic field strength responds directly and immediately to each and every one of the abrupt (1-10 min) variations in the solar wind and foreshock dynamic pressure seen by spacecraft located directly upstream of the subsolar bow shock (e.g. Sibeck et al., 1989a; Fairfield et al., 1990).

The bottom panel of Fig. 10 presents GOES-8 geosynchronous magnetic field strength observations from 16:00 to 19:00 UT on 19 April 1996. GOES-8 observed a sequence of three crater-like magnetic field strength variations, with depressions centered on 16:30, 17:00, and 18:15 UT bounded by transient magnetic field strength enhancements. Despite the similarity of these crater-like magnetic field strength structures to the density and magnetic 
field strength variations expected for foreshock cavities, there is no one-to-one relationship between the geosynchronous magnetic field strength variations and the density (or flux) variations seen by any of the solar wind monitors available on this day. We conclude that accurate predictions of the geosynchronous magnetic field require solar wind observations from a monitor located immediately upstream from the subsolar bow shock, not far off the Sun-Earth line nor far upstream at the L1 point.

\section{Summary and conclusion}

Processes within the foreshock introduce substantial variations into the incoming solar wind shortly before it interacts with the bow shock and magnetosphere. Because energetic ion fluxes should diminish exponentially and pitch angle distributions vary from isotropic to field-aligned with distance upstream, theory predicts the perturbations to attain greatest amplitudes immediately upstream from the bow shock. The observations presented in this paper confirm these predictions. Geotail, located $\sim 7 \mathrm{R}_{E}$ upstream from the nominal bow shock observed strong, and sometimes repetitive, density and magnetic field strength perturbations in antiphase with energetic ion flux variations. Interball-1, $\sim 13 \mathrm{R}_{E}$ upstream from the bow shock, observed only weak perturbations. IMP-8, $\sim 23 \mathrm{R}_{E}$ upstream from the bow shock, observed almost no foreshock related density or magnetic field strength perturbations.

The pressure of the energetic ions perpendicular to the magnetic field excavates the foreshock cavities. As theory predicts, the flux of energetic ions diminished from Geotail to IMP-8 at all energies and the energetic ion pitch angle distributions were frequently nearly isotropic at Geotail, but invariably field-aligned at IMP-8. Statistical studies have demonstrated that the e-folding distances for flux decreases range from $3.3 \mathrm{R}_{E}$ for $10 \mathrm{keV}$ ions to $11.7 \mathrm{R}_{E}$ for $67.3 \mathrm{keV}$ ions (Trattner et al., 1994), and that the transition in the pitch angle distributions of $50 \mathrm{keV}$ ions from isotropic to fieldaligned transpires within the region $15 \mathrm{R}_{E}$ upstream from the bow shock (Mitchell et al., 1983). We therefore conclude that foreshock cavities with significant amplitudes can only be observed within $\sim 10$ to $15 \mathrm{R}_{E}$ upstream from the bow shock.

This conclusion is consistent with previously reported results. Sibeck et al. (1989a) and Fairfield et al. (1990) presented several case studies in which the IRM spacecraft observed sizeable density and magnetic field strength variations $(\delta \mathrm{n} / \mathrm{n}=\delta \mathrm{B} / \mathrm{B} \sim 1)$. By contrast, Sibeck et al. (2001) reported a statistical survey of IMP- 8 observations indicating relatively minor density and magnetic field strength variations $(\delta \mathrm{n} / \mathrm{n}=\delta \mathrm{B} / \mathrm{B} \sim 0.2)$. With an apogee of $\sim 18 \mathrm{R}_{E}$, the IRM could only observe the foreshock in the immediate vicinity of the subsolar bow shock where prominent foreshock cavities are expected. IMP-8 observed the foreshock at radial distances from Earth, ranging from 30 to $40 \mathrm{R}_{E}$, where foreshock cavities should be less prominent.
The significance of the foreshock cavities lies in their ability to modify the solar wind parameters striking the dayside magnetosphere. Murr and Hughes (2003) have recently demonstrated a one-to-one relationship between foreshock cavities and the isolated mesoscale whirls of ionospheric convection seen on the edge of the polar cap and known as traveling convection vortices. Because the foreshock cavities are common and their factor of 2 to 3 density variations exceed those generally seen in the solar wind, we think it likely that they also represent a major contributor to the irreducible, and as yet inexplicable, large-amplitude fluctuations in magnetopause location about its mean position (e.g. Roelof and Sibeck, 1993). Pressure variations associated with the cavities may trigger bursts of reconnection on the dayside magnetopause (Elphic, 1992) or even generate signatures that mimic reconnection-produced flux transfer events (Sibeck, 1990), and the foreshock cavities must be a major source of transient compressions and rarefactions in the dayside magnetospheric magnetic field strength (e.g. Borodkova et al., 1995; Sanny et al., 2001). Our comparison of IMP8 , Interball-1, Geotail, and Wind observations indicates that verifying these hypotheses will either require observations in the subsolar foreshock or the ability to predict conditions in that region from observations made further upstream or off the Sun-Earth line.

Acknowledgements. We thank T. Nagai for providing high resolution Geotail magnetometer observations via the DARTS data server at ISAS, R. B. Decker for the IMP-8 CPME observations, A. Szabo for the IMP-8 GSFC magnetometer observations, A. J. Lazarus for the IMP-8 MIT plasma observations, R. W. McEntire and J. Vandegriff for the Geotail EPIC observations, R. Lin and T.-D. Phan for the Wind 3-DP plasma observations, R. P. Lepping for the Wind GSFC magnetometer observations, and S. Romanov for the Interball-1 magnetometer observations. H. J. Singer provided very helpful comments on an earlier draft of the paper. The authors also thank both referees for their comments. Many of the data sets used in this study were supplied by the NASA/GSFC CDAWeb data server. K. Kudela acknowledges support from Slovak VEGA Grant Agency Project 4064.

Topical Editor M. Lester thanks H. Lühr and another referee for their help in evaluating this paper.

\section{References}

Borodkova, N. L., Zastenker, G. N., and Sibeck, D. G.: Transient magnetic-field events at geosynchronous orbit and their solarwind origin, J. Geophys. Res., 100, 5643-5656, 1995.

Burlaga, L. F.: Micro-scale structures in the interplanetary medium, Solar Phys., 4, 67-92, 1968.

Fairfield, D. H.: Average and unusual locations of the Earth's magnetopause and bow shock, J. Geophys. Res., 76, 6700-6716, 1971.

Fairfield, D. H., Baumjohann, W., Paschmann, G., Lühr, H., and Sibeck, D. G.: Upstream pressure variations associated with the bow shock and their effects on the magnetosphere, J. Geophys. Res., 95, 3773-3786, 1990.

Gosling, J. T., Asbridge, J. R., Bame, S. J., Paschmann, G., and Sckopke, N.: Observations of two distinct populations of bow 
shock ions in the upstream solar wind, Geophys. Res. Lett., 5, 957-960, 1978.

Greenstadt, E. W., Russell, C. T., and Hoppe, M.: Magnetic field orientation and suprathermal ion streams in the Earth's foreshock, J. Geophys. Res., 85, 3473-3479, 1980.

King, J. H.: Availability of IMP-7 and IMP-8 data for the IMS period, in The IMS Source Book: Guide to the International Magnetospheric Study Data Analysis, edited by C. T. Russell and D. J. Southwood, pp. 10-20, AGU, Washington, D. C., 1982.

Klimov, S., Romanov, S., Amata, E., et al.: ASPI experiment: Measurements of fields and waves onboard the Interball-1 spacecraft, Ann. Geophys., 15, 514-527, 1997.

Kokubun, S., Yamamoto, T., Acu"ña, M. H., Hayashi, K., Shiokawa, K., and Kawano, H.: The Geotail magnetic field experiment, J. Geomagn. Geoelectr., 46, 7-21, 1994.

Lee, M. A.: Coupled hydromagnetic wave excitation and ion acceleration upstream of the Earth's bow shock, J. Geophys. Res., 87, 5063-5080, 1982.

Lepping, R. P., Acuña, M. H., Burlaga, L. F., et al.: The Wind magnetic field investigation, Space Sci. Rev., 71, 207-229, 1995.

Lin, R. P., Anderson, K. A., Ashford, S., et al.: A three-dimensional plasma and energetic particle investigation for the Wind spacecraft, Space Sci. Rev., 71, 125-153, 1995.

Lutsenko, V. N., Kudela, K., and Sarris, E. T.: The DOK-2 experiment to study energetic particles by Tail Probe and Auroral Probe satellites in the Interball project, Cos. Res. (Engl. Trans.), 36, 93-102, 1998.

Mitchell, D. G. and Roelof, E. C.: Dependence of 50-keV upstream ion events at IMP7 \&8 upon magnetic field bow shock geometry, J. Geophys. Res., 88, 5623-5634, 1983.

Mitchell, D. G., Roelof, E. C., Sanderson, T. R., Reinhard, R., and Wenzel, K.-P.: ISEE/IMP observations of simultaneous upstream ion events, J. Geophys. Res., 88, 5635-5644, 1983.

Mukai, T., Machida, S., Saito, Y., Hirahara, M., Terasawa, T., Kaya, N., Obara, T., Ejiri, M., and Nishida, A.: The low energy particle (LEP) experiment onboard the Geotail satellite, J. Geomagn. Geoelectr., 46, 669-692, 1994.

Murr, D. L. and Hughes, W. J.: Solar wind drivers of traveling convection vortices, Geophys. Res. Lett., 30, doi: 10.1029/2002GL015498, 2003.

Paschmann, G., Sckopke, N., Bame, S. J., Asbridge, J. R., Gosling, J. T., Russell, C. T., and Greenstadt, E. W.: Association of lowfrequency waves with suprathermal ions in the upstream solar wind, Geophys. Res. Lett., 6, 209-212, 1979.

Roelof, E. C. and Sibeck, D. G.: Magnetopause shape as a bivariate function of interplanetary magnetic field $B_{z}$ and solar wind dynamic pressure, J. Geophys. Res., 98, 21 421-21 450, 1993.

Safrankova, J., Zastenker, G., Nemecek, Z., Fedorov, A., Simersky, M., and Prech, L.: Small scale observation of magnetopause motion: Preliminary results of the INTERBALL project, Ann. Geophys., 15, 562-569, 1997.

Sanny, J., Berube, D., and Sibeck, D. G.: A statistical study of transient event motion at geosynchronous orbit, J. Geophys. Res., 106, 21 217-21 229, 2001.
Scholer, M., Ipavich, F. M., Gloeckler, G., Hovestadt, D., and Klecker, B.: Pitch angle distributions of energetic protons near the Earth's bow shock, Geophys. Res. Lett., 6, 707-710, 1980.

Schwartz, S. J., Chaloner, C. P., Christiansen, P. J., Coates, A. J., Hall, D. S., Johnstone, A. D., Gough, M. P., Norris, A. J., Rijnbeek, R. P., Southwood, D. J., and Woolliscroft, L. J. C.: An active current sheet in the solar wind, Nature, 318, 269-271, 1985.

Sibeck, D. G.: Transient events in the outer magnetosphere Boundary waves or flux-transfer events, J. Geophys. Res., 97, 4009-4026, 1992.

Sibeck, D. G. and Korotova, G. I., Occurrence pattterns for transient magnetic field signatures at high latitudes, J. Geophys. Res., 101, 13413-13428, 1996.

Sibeck, D. G., Baumjohann, W., Elphic, R. C., et al.: The magnetospheric response to 8-min-period strong-amplitude upstream pressure variations, J. Geophys. Res., 94, 2505-2519, 1989a.

Sibeck, D. G., Baumjohann, W., and Lopez, R. E.: Solar-wind dynamic pressure variations and transient magnetospheric signatures - Reply, Geophys. Res. Lett., 16, 1200-1202, 1989b.

Sibeck, D. G., Decker, R. B., Mitchell, D. G., Lazarus, A. J., Lepping, R. P., and Szabo, A.: Solar wind preconditioning in the flank foreshock: IMP-8 observations, J. Geophys. Res., 106, 21 675-21 688, 2001

Sibeck, D. G., Phan, T.-D., Lin, R., Lepping, R. P., and Szabo, A Wind observations of foreshock cavities: A case study, J. Geophys. Res., 107, 10.1029/2001JA007539, 2002.

Singer, H. J., Matheson, L., Grubb, R., Newman, A., and Bouwer, S. D.: Monitoring space weather with the GOES magnetometers, in GOES 8 and Beyond, edited by E. R. Washwell, Proc. SPIE Int. Soc. Opt. Eng., 2812, 299-308, 1996.

Sonnerup, B. U. O.: Acceleration of particles reflected at a shock front, J. Geophys. Res., 74, 1301-1304, 1969.

Terasawa, T.: Origin of $30 \sim 100 \mathrm{keV}$ protons observed in the upstream region of the Earth's bow shock, Planet. Space Sci., 27, 365-384, 1979.

Thomas, V. A. and Brecht, S. H.: Evolution of diamagnetic cavities in the solar wind, J. Geophys. Res., 93, 11 341-11353, 1988

Thomas, V. A., Winske, D., and Thomsen, M. F.: Simulation of upstream pressure pulse-propagation through the bow shock, J. Geophys. Res., 100, 23 481-23 488, 1995.

Trattner, K. J., Möebius, E., Scholer, M., Klecker, B., and Hilchenbach, M.: Statistical analysis of diffuse ion events upstream of the Earth's bow shock, J. Geophys. Res., 99, 13 389-13400, 1994.

Weimer, D. R., Ober, D., Maynard, N. C., Burke, W. J., Collier, M. R., McComas, D. J., Ness, N. F., and Smith, C. W.: Variable time delays in the propagation of the interplanetary magnetic field, $\mathrm{J}$ Geophys. Res., 107, doi:10.1029/2001JA009102, 2002.

Williams, D. J., McEntire, R. W., Schlemm II, C., Lui, A. T. Y., Gloeckler, G., Christon, S. P., and Gliem, F.: Geotail energetic particles and ion composition instrument, J. Geomagn. Geoelectr., 46, 39-57, 1994. 